\title{
INCLUSIONS OF INNATELY TRANSITIVE GROUPS INTO WREATH PRODUCTS IN PRODUCT ACTION WITH APPLICATIONS TO 2-ARC-TRANSITIVE GRAPHS
}

\author{
CAI-HENG LI, CHERYL E. PRAEGER, AND CSABA SCHNEIDER
}

\begin{abstract}
We study $(G, 2)$-arc-transitive graphs for innately transitive permutation groups $G$ such that $G$ can be embedded into a wreath product Sym $\Gamma$ wr $S_{\ell}$ acting in product action on $\Gamma^{\ell}$. We find two such connected graphs: the first is Sylvester's double six graph with 36 vertices, while the second is a graph with $120^{2}$ vertices whose automorphism group is Aut $\mathrm{Sp}(4,4)$. We prove that under certain conditions no more such graphs exist.
\end{abstract}

\section{INTRODUCTION}

Graphs and groups in the paper are finite; further our graphs are undirected, simple, and connected. Graphs that satisfy certain symmetry type properties have been extensively studied, especially the class of $s$-arc-transitive graphs, for $s \geqslant 2$. An $s$-arc in a graph $\Gamma$ is a sequence of vertices $\left(v_{0}, \ldots, v_{s}\right)$ such that $v_{i}$ and $v_{i+1}$ are neighbors for all $0 \leqslant i \leqslant s-1$ and $v_{i} \neq v_{i+2}$ for $0 \leqslant i \leqslant s-2$. If $G$ is a subgroup of the automorphism group of $\Gamma$ that acts transitively on the set of $s$-arcs of $\Gamma$, then we say that $\Gamma$ is $(G, s)$-arc-transitive. The graph $\Gamma$ is said to be $s$-arc-transitive, if it is (Aut $\Gamma, s$ )-arc-transitive. The values of $s$ for which this property can hold are very restricted: by the celebrated results of Weiss and Tutte, if $\Gamma$ is an $s$-arc-transitive graph with valency at least 3 , then $s \leqslant 7$ [Wei81, while if $\Gamma$ is a cubic graph then $s \leqslant 5$ [Tut47]. The classification problem of $s$-arc-transitive graphs has a long history; see for instance [Ser07] for an excellent survey.

In the theory of 2-arc-transitive graphs, normal quotients play an important role. If $\Gamma$ is a $(G, 2)$-arc-transitive graph, and $N$ is a normal subgroup of $G$ that is intransitive on the vertex set of $\Gamma$, then we construct a quotient graph $\Gamma_{N}$ whose vertices are the $N$-orbits. If the original graph $\Gamma$ is not bipartite, then $\Gamma_{N}$ is $(G / N, 2)$-arc-transitive. Further, in this case, $\Gamma$ is a normal cover of $\Gamma_{N}$; see [Pra92, Theorem 4.1] or [Ser07, Lemma 3.1]. Hence it is important to study $(G, 2)$-arc-transitive graphs that do not admit proper normal quotients. In this case, all non-trivial normal subgroups of $G$ are transitive and $G$ is said to be quasiprimitive; see [Pra92. Thus the $(G, 2)$-arc-transitive graphs for quasiprimitive permutation groups $G$ can be considered as building blocks for finite, non-bipartite, 2-arctransitive graphs, and they require special attention. However, our understanding of the class of $(G, 2)$-arc-transitive graphs is still far from complete for a general quasiprimitive groups $G$. 
Quasiprimitive groups were classified by the second author Pra92 via a type-analysis similar to the O'Nan-Scott classification of primitive groups. She showed that if $\Gamma$ is a $(G, 2)$-arc-transitive graph with a quasiprimitive group $G$, then the O'Nan-Scott type of $G$ is one of four types, denoted As, HA, PA and Tw (see BP03 for the definitions of these O'Nan-Scott types). All examples for type HA were determined in [IP93] and those of type TW were studied in [Bad93. In addition many examples of type As are known; see, for example, [FP99a, FP99b, HNP99]. On the other hand, it was not known for more than 20 years after the publication of [Pra92] whether there were examples of type PA. The first PA examples were constructed by Seress and the first author in [LS06], and links between the PA and As types were explored in a recent paper; see [LSS15, Theorem 1.3].

If a group $G$ of type PA is primitive, then the underlying set can be identified with a cartesian product $\Gamma^{\ell}$, for $\ell \geqslant 2$, in such a way that $G$ is a subgroup of the wreath product $W=\operatorname{Sym} \Gamma$ wr $S_{\ell}$ acting in product action on $\Gamma^{\ell}$; moreover $G$ has socle $T^{\ell}$, for a non-abelian simple group $T$, and the component induced by $G$ on $\Gamma$ is primitive of AS type with socle $T$. In our terminology introduced in Section 2.2, the embedding $G \leqslant W$ is a normal inclusion. It is possible, but not always the case, that an imprimitive quasiprimitive group $G$ has a normal inclusion into a wreath product in product action. However, for none of the five infinite families of examples of $(G, 2)$-arc-transitive graphs in [LS06], with $G$ quasiprimitive of type PA, does $G$ have such a normal inclusion.

In this paper we seek, and give, answers to the following questions:

(1) Are there any $(G, 2)$-arc transitive graphs, with $G$ quasiprimitive of type PA, such that $G$ has a normal inclusion in a wreath product in product action on the vertex set?

(2) More generally, do there exist any $(G, 2)$-arc transitive graphs with $G$ quasiprimitive such that $G$ can be embedded in a wreath product in product action on the vertex set? In particular, is this true for any of the examples in [LS06]?

Our results answer both questions, yet raise an interesting open problem. First we give a negative answer to Question 1 (Corollary 3.5), and in Section 6 we analyze the examples in [LS06] and prove (Corollary 6.4) that for none of them does the group $G$ embed in a wreath product in product action. The latter conclusion relies on our general analysis of the situation for Question 2. Our main result Theorem 1.1 proves that there do indeed exist at least two examples, with each of these examples having type As, that is the group $G$ is almost simple.

Using the terminology of [Pra90, BP03], we say that the containment $G \leqslant W$ is an inclusion of $G$ into $W$, and if $W=\operatorname{Sym} \Delta \mathrm{wr} S_{\ell}$ in product action on $\Delta^{\ell}$, then $W$ is the full stabilizer of the natural cartesian decomposition of $\Delta^{\ell}$ (see Section 2), and hence a subgroup $G$ of $W$ also stabilizes this decomposition. Our analysis for Question 2 builds on the theory developed by the second and third authors in a series of papers [BPS04, BPS06, BPS07, PS07, BPS08, PS12] with Baddeley to describe the cartesian decompositions stabilized by a given innately transitive group, namely a permutation group with a transitive minimal normal subgroup $N$. Such a subgroup $N$ is called a plinth. Since each 
quasiprimitive group is innately transitive, we make our analysis in the broader context of innately transitive groups. Moreover, since the case of abelian plinths is completely settled in Proposition 3.6 using the results in [IP93, we usually assume that the plinth is non-abelian. Further, since for quasiprimitive type PA the plinth is not regular, we assume this also. The type $\mathrm{CD}_{2 \nsim}$ for an inclusion $G \leqslant W$ is defined in Section 2.3 .

Theorem 1.1. Suppose that $\Delta$ is a finite set with $|\Delta| \geqslant 2$ and let $\ell \geqslant 2$. Consider $W=\operatorname{Sym} \Delta w r S_{\ell}$ as a permutation group acting in product action on $\Omega=\Delta^{\ell}$. Let $\Gamma$ be a connected $(G, 2)$-arc-transitive graph with vertex set $\Omega$ for some innately transitive group $G \leqslant W$ with non-regular plinth $M$. Then one of the following is valid.

(1) $G$ is quasiprimitive of type As and either

(a) $M \cong \mathrm{A}_{6}, \mid$ Aut $\mathrm{A}_{6}: G|\in\{1,2\}, G \neq \mathrm{PGL}(2,9),| \Omega \mid=6^{2}$, and $\Gamma$ has valency 5 ; or

(b) $M \cong \mathrm{Sp}(4,4), G=$ Aut $\mathrm{Sp}(4,4),|\Omega|=120^{2}$ and $\Gamma$ is a graph with valency 17 .

(2) $M$ non-abelian and non-simple, $G$ projects onto a transitive subgroup of $\mathrm{S}_{\ell}$ and the inclusion $G \leqslant W$ is of type $\mathrm{CD}_{2 \nsim}$.

There is a unique example for each part of Theorem 1.1(1). These two graphs are, to our knowledge, the first known connected $(G, 2)$-arc transitive graphs with $G$ quasiprimitive and preserving a cartesian decomposition of the vertex set. The graph in part (a) is known as Sylvester's Double Six Graph (see [BCN89, 13.1.2 Theorem]). Both graphs in part (1) can be constructed using the generalized quadrangle associated with the non-degenerate alternating bilinear form preserved by $\operatorname{Sp}(4, q)$ with $q=2,4$. On the other hand, the existence of examples in part (2) is unresolved.

Problem. Does there exist a $(G, 2)$-arc transitive graph $\Gamma$ for which Theorem $1.1(2)$ holds?

The class of inclusions of type $C_{2} \nsim$ is discussed in Section 2.3. Of the six types of inclusions characterized there, it is the one for which least information is available. So it is not surprising that for these inclusions our results are, unfortunately, inconclusive. The theory of cartesian decompositions invariant under the action of an innately transitive group is spread over several papers, and is presented in terms of cartesian systems of subgroups of the plinth. We found that the language of inclusions into wreath products was better suited for our study of 2-arc-transitive graphs, and we believe that this is probably true also for other applications in algebraic combinatorics and graph theory. For this reason we summarize the most important aspects of the theory in Section 2 using the language of inclusions. In particular, if $G \leqslant W=\operatorname{Sym} \Delta w r S_{\ell}$, acting in product action on $\Delta^{\ell}$, with $G$ innately transitive, then the image $G \pi$ under the natural projection map $\pi: W \rightarrow \mathrm{S}_{\ell}$ has at most two orbits on $\underline{\ell}=\{1, \ldots, \ell\}$. The groups that have two orbits can be characterized, and the groups that are transitive on $\underline{\ell}$ are classified into six classes. For all classes except for $C_{2}$, the class that appears in Theorem $1.1(2)$, the information that we have about $G$ is so strong that its permutational isomorphism type can be described in quite precise details (see Theorem 2.9). The results from Section 2 
are applied in Sections 36 6 to prove Theorem 1.1 and to analyze the graphs in [LS06]. We believe that this summary will make the results more accessible for other researchers. For example, the study by Morris and Spiga in MS12 significantly generalized results about distance-transitive graphs to $(G, \Lambda)$-transitive digraphs for which the group $G$ is innately transitive and has a normal inclusion into a wreath product in product action. Perhaps using the framework we present in Section 2, their results could be further extended to describe not necessarily normal inclusions for such graphs.

\section{INCLUSIONS OF INNATELY TRANSITIVE GROUPS INTO WREATH PRODUCTS}

We introduce notation that we will keep throughout the section. For a natural number $n$, the symbol $\underline{n}$ denotes the set $\{1, \ldots, n\}$. We will work under the following hypothesis.

Hypothesis 2.1. Suppose that $\Delta$ is a finite set with $|\Delta| \geqslant 2, \ell \geqslant 2$, and let $\Omega=\Delta^{\ell}$. Consider $W=\operatorname{Sym} \Delta w r S_{\ell}=(\operatorname{Sym} \Delta)^{\ell} \rtimes S_{\ell}$ as a permutation group acting in product action on $\Omega$. Suppose that $G$ is subgroup of $W$ and $M$ is a minimal normal subgroup of $G$ that is transitive on $\Omega$. Let $M=T_{1} \times \cdots \times T_{k} \cong T^{k}$ where the $T_{i}$ and $T$ are finite simple groups.

By the definition introduced in the introduction, $G$ is an innately transitive group on $\Omega$ with plinth $M$. For $\left(\delta_{1}, \ldots, \delta_{\ell}\right) \in \Omega, g_{1}, \ldots, g_{\ell} \in \operatorname{Sym} \Delta$ and $h \in \mathrm{S}_{\ell}$ the product action of $\left(g_{1}, \ldots, g_{\ell}\right) h \in W$ on $\left(\delta_{1}, \ldots, \delta_{\ell}\right)$ can be written as

$$
\left(\delta_{1}, \ldots, \delta_{\ell}\right)\left(g_{1}, \ldots, g_{\ell}\right) h=\left(\delta_{1 h^{-1}} g_{1 h^{-1}}, \ldots, \delta_{\ell h^{-1}} g_{\ell h^{-1}}\right) .
$$

Inclusions of primitive and quasiprimitive groups into wreath products in product action were described in [Pra90, BP03]. Innately transitive subgroups of wreath products in product action were studied in [BPS04, BPS06, BPS07, PS07, BPS08, by analyzing cartesian decompositions. A cartesian decomposition of $\Omega$ is a set $\mathcal{E}=\left\{\Delta_{1}, \ldots, \Delta_{\ell}\right\}$ of partitions of $\Omega$ such that

$$
\left|\delta_{1} \cap \cdots \cap \delta_{\ell}\right|=1 \quad \text { for all } \quad \delta_{1} \in \Delta_{1}, \ldots, \delta_{\ell} \in \Delta_{\ell} .
$$

In our case $W$ is the full stabilizer in Sym $\Omega$ of the natural cartesian decomposition $\mathcal{E}$ of $\Omega$ where $\mathcal{E}$ is defined by

$$
\mathcal{E}=\left\{\Delta_{i} \mid i \in \underline{\ell}\right\} \quad \text { where } \quad \Delta_{i}=\left\{\left\{\left(\delta_{1}, \ldots, \delta_{\ell}\right) \mid \delta_{i}=\delta\right\} \mid \delta \in \Delta\right\}
$$

(see [PS12, Section 1.1]). The cartesian decomposition $\mathcal{E}$ contains $\ell$ partitions of $\Omega$, and $\left(\delta_{1}, \ldots, \delta_{\ell}\right)$ and $\left(\gamma_{1}, \ldots, \gamma_{\ell}\right)$ belong to the same block of $\Delta_{i}$ if and only if $\delta_{i}=\gamma_{i}$. The group $W$ preserves $\mathcal{E}$ in the sense that it permutes the partitions in $\mathcal{E}$.

The aim of this section is to combine the theory of cartesian decompositions with the Embedding Theorem in PS12 to derive useful facts on the inclusion of $G$ into $W$ that can be used in the characterization of $(G, 2)$-arc-transitive graphs in Sections 3 5. The main results do not refer to $\mathcal{E}$ directly, but the fact that $W$ and $G$ preserve $\mathcal{E}$ plays a central role in their proofs. For the key assertions we give references to the appropriate results in the papers cited above. 
Suppose that $\pi: W \rightarrow \mathrm{S}_{\ell}$ is the natural projection and consider $\pi$ as a permutation representation of $W$ on $\underline{\ell}$. The kernel of $\pi$ is the base group (Sym $\Delta)^{\ell}$ of the wreath product $W$. If $i, j \in \underline{\ell}$, then we have, for all $w \in W$, that $i(w \pi)=j$ if and only if $\Delta_{i} w=\Delta_{j}$. Thus the $W$-actions on $\underline{\ell}$ and on $\mathcal{E}$ are permutationally equivalent. This permutational equivalence implies that the pointwise stabilizer in $W$ of $\mathcal{E}$ is the base group (Sym $\Delta)^{\ell}$ of the wreath product. Combining this with [BPS04, Proposition 2.1] gives the following.

Lemma 2.2. Assuming Hypothesis 2.1, $M \leqslant(\operatorname{Sym} \Delta)^{\ell}$.

In [PS12, we defined, for $j \in \underline{\ell}$, the $j$-th component of the group $G$. Let us express this component in terms of the inclusion $G \leqslant W$. Let $W_{j}$ denote the stabilizer of $j$ under $\pi$. The group $W_{j}$ can be written as a direct product

$$
W_{j}=\operatorname{Sym} \Delta \times\left(\operatorname{Sym} \Delta \mathrm{wr} \mathrm{S}_{\ell-1}\right)
$$

where the first factor acts on the $j$-th coordinate of $\Omega=\Delta^{\ell}$ and the second factor acts on the other $\ell-1$ coordinates. In particular ' $\mathrm{S}_{\ell-1}$ ' is meant to be the stabilizer of $j$ in $\mathrm{S}_{\ell}$. We define the $j$-th component $G^{(j)}$ of $G$ as the projection of $G_{j}=G \cap W_{j}$ into the first factor of the direct product decomposition of $W_{j}$ given in (1). It is easy to see that this definition of the component is equivalent to the one in [PS12, Section 1.2].

As both $G$ and $M$ are transitive on $\Omega$, the following is a direct consequence of Theorems 1.1-1.2 of [PS12].

Theorem 2.3. Suppose that Hypothesis 2.1 is valid and let $\omega \in \Omega$.

(1) The components $G^{(j)}$ and $M^{(j)}$ are transitive on $\Delta$ for all $j \in \underline{\ell}$.

(2) There is an element $x \in(\operatorname{Sym} \Delta)^{\ell}$ stabilizing $\omega$ such that the components of $G^{x}$ are constant on each $G \pi$-orbit in $\underline{\ell}$.

(3) If $G \pi$ is transitive on $\underline{\ell}$ then the element $x$ in part (2) can be chosen so that $G^{x} \leqslant G^{(1)} \operatorname{wr}(G \pi)$.

The inclusion of $G$ into $W$ can be further studied by understanding the components of $M$. When $M$ is abelian, then $G$ is primitive of HA type. The fact that this case does not occur for a $(G, 2)$-arc-transitive graph is proved in Proposition 3.6 using the tools developed for primitive inclusions in [Pra90]. Thus from now on we assume that $M$ is non-abelian.

2.1. Non-abelian $\boldsymbol{M}$. Suppose that $M$ is non-abelian. In this case, $M=T^{k}=T_{1} \times \cdots \times T_{k}$ where the $T_{i}$ are the minimal normal subgroups of $M$. The $j$-th component $M^{(j)}$ is, by definition, a quotient of $M$. Suppose that $\overline{M^{(j)}}$ is the kernel of the natural projection $M \rightarrow M^{(j)}$. Then $\overline{M^{(j)}}$ is the direct product of some of the $T_{i}$. We will identify $M^{(j)}$ with the product of the $T_{i}$ such that $T_{i} \nless \overline{M^{(j)}}$. Hence, $M=\overline{M^{(j)}} \times M^{(j)}$ and $M^{(j)}$ acts faithfully on $\Delta$ viewed as the $j$-th coordinate of $\Delta^{\ell}$. Let $T$ be a simple factor of $M$ and set $\bar{T}=\mathrm{C}_{M}(T)$, so that $M=T \times \bar{T}$. If $H$ is a subgroup of $M$, then $H \bar{T} / \bar{T}$ is the projection of $H$ into $T$, and we identify this quotient with a subgroup of $T$. The following lemma states important factorization properties of the projections $\left(M^{(j)}\right)_{\delta} \bar{T} / \bar{T}$, where $\delta \in \Delta$, for those components $\left(M^{(j)}\right)$ that contain $T$. 
Lemma 2.4. Suppose that Hypothesis 2.1 holds, that $M$ is non-abelian, and that $T$ is a simple factor of $M$. Suppose that $T$ is contained in the components $M^{\left(j_{1}\right)}, \ldots, M^{\left(j_{s}\right)}$. Let $\delta \in \Delta$, set $\omega=(\delta, \ldots, \delta)$, and for $r \in \underline{s}$, let $A_{r}=\left(M^{\left(j_{r}\right)}\right)_{\delta} \bar{T} / \bar{T}$. Then

$$
A_{r}\left(\bigcap_{i \neq r} A_{i}\right)=T \text { for all } r \text { and } M_{\omega} \bar{T} / \bar{T} \leqslant \bigcap_{r \leqslant s} A_{r} \text {. }
$$

Proof. We introduced, in [BPS04, a cartesian system of subgroups associated with a cartesian decomposition. The cartesian system of subgroups with respect to $\omega=(\delta, \ldots, \delta) \in \Omega$ for the natural cartesian decomposition $\mathcal{E}$ of $\Omega=\Delta^{\ell}$ is defined as $\left\{K_{1}, \ldots, K_{\ell}\right\}$ where $K_{j}$ is the stabilizer in $M$ of the set

$$
\left\{\left(\delta_{1}, \ldots, \delta_{\ell}\right) \mid \delta_{j}=\delta\right\} .
$$

Simple consideration shows that $K_{j}=\left(M^{(j)}\right)_{\delta} \times \overline{M^{(j)}}$. Now the result follows by applying the projection $M \rightarrow T$ to the equations in [BPS04, Definition 1.3]. qed

The information given by the previous lemma is very strong. Using the terminology of [BP98, the set of proper subgroups among $A_{1}, \ldots, A_{s}$ forms a strong multiple factorization for the non-abelian finite simple group $T$. The fact that we understand such factorizations in sufficient details leads to the structure theorem in Section 2.4.

2.2. Normal inclusions. Assume that $M$ is non-abelian and identify each projection $M^{(j)}$ with a normal subgroup of $M$ as above. The inclusion $G \leqslant W$ is said to be normal if $M=\prod_{j} M^{(j)}$. Since our definition of the component $M^{(j)}$ is equivalent to the one given in [BPS07, an inclusion $G \leqslant W$ is normal if and only if the natural cartesian decomposition $\mathcal{E}$ of $\Delta^{\ell}$ is $M$-normal, as defined in [BPS07]. The inclusion $G \leqslant W$ is normal if and only if for all $T_{i}$ there is a unique $j$ such that $T_{i} \leqslant M^{(j)}$; that is, each $T_{i}$ acts trivially on all but one coordinate of $\Delta^{\ell}$. The following proposition is a direct consequence of [BPS07, Lemma 3.1].

Proposition 2.5. Suppose Hypothesis 2.1 and let $\omega=(\delta, \ldots, \delta)$ with some $\delta \in \Delta$. If $G \leqslant W$ is a normal inclusion, then $G \pi$ is transitive on $\underline{\ell}$ and $M_{\omega}=\prod_{j}\left(M^{(j)}\right)_{\delta}$.

The following result gives a criterion to recognize possible normal inclusions $G \leqslant W$. For a group $X$, a set $\mathcal{X}=\left\{X_{1}, \ldots, X_{r}\right\}$ of subgroups of $X$ is said to be a direct decomposition of $X$ if $X=X_{1} \times \cdots \times X_{r}$. A subgroup $Y \leqslant X$ is said to be an $\mathcal{X}$ subgroup, if $\left\{Y \cap X_{i} \mid i \in \underline{r}\right\}$ is a direct decomposition of $Y$. When $G \leqslant W$ is a normal inclusion, then $M_{\omega} \cap M^{(j)}=\left(M^{(j)}\right)_{\delta}$, and so Proposition 2.5 states that $\mathcal{X}=\left\{M^{(j)} \mid j \in \underline{\ell}\right\}$

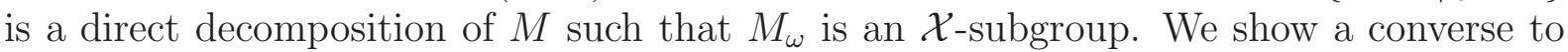
this statement in the next theorem.

Theorem 2.6. Suppose that $G$ is a finite innately transitive permutation group acting on $\Omega$ with a non-abelian plinth $M$ and let $\omega \in \Omega$. Suppose that there exists a $G$-invariant direct decomposition $\mathcal{X}=\left\{M_{1}, \ldots, M_{r}\right\}$ of $M$ such that $M_{\omega}$ is an $\mathcal{X}$-subgroup. Let $\Xi$ be the right coset space $\left[M_{1}: M_{1} \cap M_{\omega}\right]$. Then there exists a monomorphism $\alpha: G \rightarrow \operatorname{Sym} \Xi \mathrm{wr}_{r}$, 
where the wreath product is taken as a permutation group acting in product action on $\Xi^{r}$, in such a way that the inclusion $G \alpha \leqslant$ Sym $\Xi \mathrm{wr}_{r}$ is a normal inclusion.

Proof. Given a direct product decomposition of $M$ as above, one can construct a normal cartesian decomposition $\mathcal{E}^{\prime}$ preserved by $G$ as in [BPS06, Example 7.1]. The full stabilizer of $\mathcal{E}^{\prime}$ is isomorphic to Sym $\Xi w r S_{r}$ in product action (see [PS12, Section 1.2]) and $G$ can be embedded into this wreath product using a monomorphism $\alpha$. Since $\mathcal{E}^{\prime}$ is $M$-normal, the inclusion $G \alpha \leqslant \operatorname{Sym} \Xi \mathrm{wrS}_{r}$ is normal. qed

In the case when $G$ is primitive, the direct product decomposition $\left\{M_{1}, \ldots, M_{r}\right\}$ in the theorem is referred to as a blow-up decomposition by [Kov89.

Lemma 2.7. Assuming Hypothesis 2.1, if $G \leqslant W$ is a normal inclusion, then $M^{(j)}$ is a transitive minimal normal subgroup of $G^{(j)}$. Further, if $M$ is non-regular, $M^{(j)}$ is the unique such transitive minimal normal subgroup of $G^{(j)}$.

Proof. Since $M \unlhd G, M^{(j)} \unlhd G^{(j)}$. Theorem 2.3 implies that $M^{(j)}$ is transitive. Since $M$ is non-abelian, to prove that $M^{(j)}$ is a minimal normal subgroup of $G^{(j)}$, it suffices to show that $G^{(j)}$ permutes transitively the simple components of $M^{(j)}$. Suppose, without loss of generality, that $T_{1}$ and $T_{2}$ are simple components of $M^{(j)}$. Then there is some $g \in G$ such that $\left(T_{1}\right)^{g}=T_{2}$. Since $G \leqslant W$ is a normal inclusion, $M^{(j)}$ is the unique component that contains $T_{1}$ and $T_{2}$. Hence, $g \pi \in W_{j}$. Thus, using the decomposition in (1), $g=g_{1} g_{2}$ where $g_{1} \in G^{(j)}$ and $g_{2} \in(\operatorname{Sym} \Delta)$ wr $\mathrm{S}_{\ell-1}$. The component $g_{2}$ centralizes both $T_{1}$ and $T_{2}$, and hence we must have that $\left(T_{1}\right)^{g_{1}}=T_{2}$. Thus $G^{(j)}$ is transitive on the simple components of $M^{(j)}$, and hence $M^{(j)}$ is a minimal normal subgroup of $G^{(j)}$.

By Proposition 2.5,

$$
|\Omega|=\left|M: M_{\omega}\right|=\prod_{j=1}^{\ell}\left|M^{(j)}:\left(M^{(j)}\right)_{\delta}\right|=\frac{M}{\prod_{j}\left|\left(M^{(j)}\right)_{\delta}\right|} .
$$

Hence $M$ is regular if and only if each component $M^{(j)}$ is regular. In particular, if $M$ is not regular, then neither is $M^{(j)}$ and then it must be the unique minimal normal subgroup of $G^{(j)}$. qed

2.3. Transitive $\boldsymbol{G} \boldsymbol{\pi}$. We now turn our attention to non-normal inclusions. Assume that $G \pi$ is transitive on $\underline{\ell}$. This implies that the conjugation action of $G$ is transitive on the set $\left\{M^{(j)}\right\}$. Set $T=T_{1}$. Since $G$ acts transitively on the $T_{i}$ by conjugation, the treatment that follows does not depend on this choice of $T$. The group $T$ can be a subgroup in several of the components $M^{(j)}$. Since $G$ acts transitively on both sets $\left\{T_{i}\right\}$ and $\left\{M^{(j)}\right\}$, the number of components $M^{(j)}$ such that $T \leqslant M^{(j)}$ is independent of the choice of $T$. The inclusion $G \leqslant W$ is normal if and only if this number is one. We will investigate here the situation when this is not the case; that is, $T$ is contained in at least two $M^{(j)}$. As above, we set $\bar{T}=\mathrm{C}_{M}(T)$.

Theorem 2.8. Suppose that Hypothesis 2.1 holds, that $M$ is non-abelian, and that $G \pi$ is transitive. Suppose that $T$ is a simple factor of $M$. Then the following are valid. 
(1) The number $s$ of components $M^{(j)}$ such that $T \leqslant M^{(j)}$ is independent of the choice of $T$ and $s \leqslant 3$.

(2) The inclusion $G \leqslant W$ is normal if and only if $s=1$.

(3) If $\left(M^{(j)}\right)_{\delta} \bar{T} / \bar{T}=T$ for some $j$ and $\delta \in \Delta$ then $s \leqslant 2$.

Proof. As explained in the proof of Lemma 2.4, the cartesian system of subgroups with respect to $\omega=(\delta, \ldots, \delta)$ that corresponds to the natural cartesian decomposition of $\Omega$ is $\left\{K_{1}, \ldots, K_{\ell}\right\}$ where $K_{j}=\left(M^{(j)}\right)_{\delta} \times \overline{M^{(j)}}$. Now parts (1) and (3) follow from [BPS06, Theorem 6.1]. Part (2) was justified before the theorem. qed

We use Theorem 2.8 to define the inclusion types $C_{1 S}, C_{2 \sim}, C_{2 \nsim}$, and $C_{3}$ for inclusions $G \leqslant W$ in Hypothesis 2.1 such that $T$ is non-abelian and $G \pi$ is transitive on $\underline{\ell}$. If, in Theorem 2.8, $s=1$, the inclusion $G \leqslant W$ is normal, and so we assume that this is not the case. Set $\delta \in \Delta$.

$\mathbf{C D}_{1 S}$ : We say that the inclusion $G \leqslant W$ is of type $\mathrm{CD}_{1 S}$ if $s=2$ and $\left(M^{(j)}\right)_{\delta} \bar{T} / \bar{T}=T$ for some $j$.

$\mathbf{C D}_{2}$ : Suppose that $s=2$ and $\left(M^{(j)}\right)_{\delta} \bar{T} / \bar{T} \neq T$ for all $j$. Let $j_{1}$ and $j_{2}$ be distinct indices such that $T \leqslant M^{\left(j_{1}\right)} \cap M^{\left(j_{2}\right)}$. Then the coordinate projections $A=\left(M^{\left(j_{1}\right)}\right)_{\delta} \bar{T} / \bar{T}$ and $B=\left(M^{\left(j_{2}\right)}\right)_{\delta} \bar{T} / \bar{T}$ are proper subgroups of $T$. We say that the inclusion $G \leqslant W$ is of type $\mathrm{CD}_{2 \sim}$ if $A \cong B$. Otherwise the inclusion is of type $\mathrm{CD}_{2 \varkappa}$.

$\mathbf{C D}_{3}$ : We say that the inclusion $G \leqslant W$ is of type $\mathrm{CD}_{3}$ if $s=3$.

The fact that these types are well-defined was proved in [BPS06, Theorem 6.2]. The definition of the inclusion type $\mathrm{CD}_{2 \sim}$ is slightly different in [BPS06] where we required that the two proper projections should actually be $G_{\omega}$-conjugates. The definition given here leads to a stronger theorem.

Suppose that $G \leqslant W$ is an inclusion of type $\mathrm{CD}_{1 S}$ and fix $\delta \in \Delta$. Suppose that $T$ is a simple factor of $M$ such that $T \leqslant M^{(j)}$ and $\left(M^{(j)}\right)_{\delta} \bar{T} / \bar{T}=T$. By a version of Scott's Lemma [BPS06, Lemma 4.2] on subdirect subgroups in direct products of nonabelian simple groups, $\left(M^{(j)}\right)_{\delta}$ contains a full diagonal subgroup isomorphic to $T$. Then $\left(M^{(j)}\right)_{\delta}=D \times \mathrm{C}_{\left(M^{(j)}\right)_{\delta}}(D)$ where $D$ is a full diagonal subgroup in $T \times T_{i}$ for some $i$; that is, $D=\{(t, t \alpha) \mid t \in T\}$ with some isomorphism $\alpha: T \rightarrow T_{i}$. We say that the full diagonal subgroup $D$ is involved in $\left(M^{(j)}\right)_{\delta}$ and that $\left\{T, T_{i}\right\}$ is the support of $D$. In this case, $k$ is even and $M$ admits a direct decomposition

$$
\mathcal{S}=\left\{M_{1}, \ldots, M_{k / 2}\right\}
$$

such that $M_{i}=T_{i_{1}} \times T_{i_{2}}$ where $\left\{T_{i_{1}}, T_{i_{2}}\right\}$ is the support of some full diagonal subgroup $D$ involved in $\left(M^{(j)}\right)_{\delta}$ for some $j$; see [BPS06, Theorem 6.1].

2.4. A structure theorem. The power of characterizing inclusions $G \leqslant W$ into types as above lies in the fact that in many cases strong information can be deduced about the permutational isomorphism type of $M$. We denote the dihedral group of order $2 a$ by $\mathrm{D}_{a}$. 


\begin{tabular}{|c|c|c|c|c|c|c|c|c|}
\hline & 1 & 2 & 3 & 4 & 5 & 6 & 7 & 8 \\
\hline$T$ & $\mathrm{~A}_{6}$ & $\begin{array}{c}\mathrm{Sp}\left(4,2^{a}\right) \\
a \geqslant 2\end{array}$ & $\mathrm{P}^{+}(8, q)$ & $\mathrm{M}_{12}$ & $\mathrm{M}_{12}$ & $\begin{array}{c}\mathrm{Sp}(4 a, 2) \\
a \geqslant 2\end{array}$ & $\mathrm{P}^{+}(8,3)$ & $\mathrm{Sp}(6,2)$ \\
\hline$X$ & $\mathrm{D}_{10}$ & $\mathrm{D}_{2^{a}+1}$ & $\mathrm{G}_{2}(q)$ & $\mathrm{PSL}(2,11)$ & $C_{11} \rtimes C_{5}$ & $C_{2} \times \mathrm{Sp}(2 a-2,4)$ & $C_{6} \rtimes \mathrm{S}_{4}$ & $\mathrm{D}_{3}$ \\
\hline$Y$ & $\mathrm{D}_{10}$ & $\mathrm{D}_{2^{a}+1} \cdot 2$ & $\mathrm{G}_{2}(q)$ & $\mathrm{PSL}(2,11)$ & $C_{11} \rtimes C_{5}$ & $C_{2} \times \mathrm{Sp}(2 a-2,4)$ & $C_{6} \rtimes \mathrm{S}_{4}$ & $\mathrm{D}_{3} \cdot 2$ \\
\hline
\end{tabular}

TABLE 1. Table for Theorem 2.9

Theorem 2.9. Assume that Hypothesis 2.1 holds, that $M$ is non-abelian and let $T$ be a simple factor of $M$. If the inclusion type is $\mathrm{CD}_{1 S}$ then let $\mathcal{S}$ be the direct decomposition of $M$ as in (2); otherwise set $\mathcal{S}=\left\{T_{1}, \ldots, T_{k}\right\}$. Set $k_{1}=|\mathcal{S}|$ and let $\omega \in \Omega$.

(1) $G \pi$ has at most two orbits in $\underline{\ell}$. Further, if $G \pi$ has two orbits in $\underline{\ell}$ then $T$ is isomorphic to one of the groups in columns 1-5 of Table 1 .

(2) If the inclusion $G \leqslant W$ has type $\mathrm{CD}_{1 S}$ or $\mathrm{CD}_{2 \sim}$, then $T$ is isomorphic to one of the groups in columns 1-4 of Table 1 .

(3) If the type of the inclusion $G \leqslant W$ is $\mathrm{CD}_{3}$, then $T$ is isomorphic to one of the groups in columns $6-8$ of Table 1 .

(4) In all parts (1)-(3), we have that $M_{\omega}$ contains an $\mathcal{S}$-subgroup isomorphic to $X^{k_{1}}$ and is contained in an $\mathcal{S}$-subgroup isomorphic to $Y^{k_{1}}$ where $X$ and $Y$ are as in the corresponding column of Table 1 . In particular, when $X=Y$, then $M_{\omega}$ is an $\mathcal{S}$-subgroup.

(5) If the inclusion $G \leqslant W$ has type $\mathrm{CD}_{2 \varkappa}$, then $T$ can be factorized as $T=A B$ with proper subgroups $A, B \leqslant T$ and $M_{\omega}$ is isomorphic to a subgroup of $(A \cap B)^{k}$.

Proof. Using the observations on the cartesian system of subgroups in the proof of Theorem 2.4, the claims of this theorem are simple consequences of the results published in [PS03, BPS06, PS07, BPS08]. (1) follows from Theorems 8.1 and 8.2 of [BPS08]. (2)-(3) follow from [BPS06, Theorem 6.3]. (4) follows from Theorem 6 of [PS03, Proposition 5.2, Theorem 7.1 and Proposition 7.2 of [PS07]. Claim (5) follows from Lemma 2.4. qed

When $M=T$ is non-abelian simple, then an inclusion $G \leqslant W$ as in Hypothesis 2.1 such that $G \pi$ is transitive must have type $C_{2 \sim}$ (see [BPS06, Theorem 6.2]). It is an important consequence of Theorem 2.6 that in all of the cases of Theorem 2.9 when $M_{\omega}$ is an $\mathcal{S}$-subgroup with $k_{1} \geqslant 2$, there is also a normal inclusion $G \leqslant \operatorname{Sym} \Xi \mathrm{wr}_{k_{1}}$.

\section{Cartesian Decompositions and arC-Transitive graphs}

We will work under the following assumptions.

Hypothesis 3.1. Assume Hypothesis 2.1 and, in addition, that $\Gamma$ is a connected graph with vertex set $\Omega$ and that $G$ is a subgroup of Aut $\Gamma$.

By Lemma 2.2, $M \leqslant(\operatorname{Sym} \Delta)^{\ell}$. We denote by $\mathrm{V}(\Gamma)$ and $\mathrm{E}(\Gamma)$ the vertex set and the edge set of $\Gamma$, respectively. For $\alpha \in \mathrm{V}(\Gamma)$, we let $\Gamma(\alpha)$ denote the neighborhood 
$\{\beta \mid\{\alpha, \beta\} \in \mathrm{E}(\Gamma)\}$ of $\alpha$ in $\Gamma$. Then the stabilizer $G_{\alpha}$ acts on $\Gamma(\alpha)$. As $G$ is assumed to be transitive on $\mathrm{V}(\Gamma)$, the graph $\Gamma$ is $(G, 2)$-arc-transitive if and only if $G_{\alpha}$ is 2-transitive on $\Gamma(\alpha)$.

Lemma 3.2. Suppose that Hypothesis 3.1 holds, that $M$ is non-regular and that $G_{\alpha}$ is quasiprimitive on $\Gamma(\alpha)$. Then $\Gamma$ is $M$-arc-transitive, and so $M_{\alpha}$ is transitive on $\Gamma(\alpha)$ for all $\alpha \in \mathrm{V}(\Gamma)$.

Proof. As $M_{\alpha} \unlhd G_{\alpha}$, either $M_{\alpha}$ is transitive or $M_{\alpha}$ is trivial on $\Gamma(\alpha)$. Suppose that $M_{\alpha}$ is trivial. We show that in this case $M_{\alpha}$ fixes all vertices of $\Gamma$, which is impossible as $M$ is not regular. We have by the conditions that $M_{\alpha}$ fixes all vertices in $\{\alpha\} \cup \Gamma(\alpha)$; that is, it fixes all vertices of distance less than or equal to 1 from $\alpha$. Thus if $\beta \in \Gamma(\alpha)$, then $M_{\beta}=M_{\alpha}$ and $M_{\beta}$ fixes all vertices of $\Gamma(\beta)$. Thus $M_{\alpha}$ fixes all vertices with distance at most 2 from $\alpha$. Continuing by induction and using that $\Gamma$ is connected, $M_{\alpha}$ fixes all vertices of $\Gamma$. This is a contradiction, and hence $M_{\alpha}$ is transitive on $\Gamma(\alpha)$. qed

Suppose that $\pi: W \rightarrow \mathrm{S}_{\ell}$ is the natural projection, and, for $j \in \underline{\ell}$, let $G^{(j)}$ be the component as defined in Section 2. If $G \pi$ is transitive, then we may assume, by Theorem [2.3, that $G \leqslant G^{(1)} \operatorname{wr}(G \pi)$.

Lemma 3.3. Assume Hypothesis [3.1, that $\Gamma$ is $M$-arc-transitive, that $G \pi$ is transitive on $\underline{\ell}$ and that $G \leqslant G^{(1)} \operatorname{wr}(G \pi)$. Let $\alpha=\left(\alpha_{1}, \ldots, \alpha_{1}\right) \in \Omega, \beta=\left(\beta_{1}, \ldots, \beta_{\ell}\right) \in \Gamma(\alpha)$ and set $K=G^{(1)}$. Then there is some $h \in\left(K_{\alpha_{1}}\right)^{\ell}$ such that $\beta h=\left(\beta_{1}, \ldots, \beta_{1}\right)$. Thus, in the image graph $\Gamma h$, the tuples $\left(\alpha_{1}, \ldots, \alpha_{1}\right)$ and $\left(\beta_{1}, \ldots, \beta_{1}\right)$ are connected. Furthermore, $G^{h} \leqslant$ Aut $(\Gamma h)$ with $G^{h} \leqslant G^{(1)}$ wr $(G \pi)$.

Proof. Since $M_{\alpha}$ is transitive on $\Gamma(\alpha)$ and $M_{\alpha} \leqslant\left(K_{\alpha_{1}}\right)^{\ell}$, we obtain that

$$
\Gamma(\alpha) \subseteq \beta_{1} K_{\alpha_{1}} \times \cdots \times \beta_{\ell} K_{\alpha_{1}}
$$

where $\beta_{i} K_{\alpha_{1}}$ is the $K_{\alpha_{1}}$-orbit containing $\beta_{i}$ and the product is the cartesian product of sets. We claim that $\beta_{1} K_{\alpha_{1}}=\beta_{i} K_{\alpha_{1}}$ for all $i$. Choose $i \in\{1, \ldots, \ell\}$. Since $M$ is transitive on $\Omega, G=M G_{\alpha}$. Since $G \pi$ is transitive on $\ell$ and, by Lemma 2.2, $M$ is in the kernel of $\pi, G_{\alpha} \pi$ must be transitive on $\underline{\ell}$. Thus there is $g \in G_{\alpha}$ such that $g=\left(h_{1}, \ldots, h_{\ell}\right) \sigma$ with $h_{1}, \ldots, h_{\ell} \in K_{\alpha_{1}}, \sigma \in G \pi$ and $1 \sigma=i$. Now

$$
\beta g=\left(\beta_{1}, \ldots, \beta_{\ell}\right)\left(h_{1}, \ldots, h_{\ell}\right) \sigma=\left(\beta_{1} h_{1}, \ldots, \beta_{\ell} h_{\ell}\right) \sigma .
$$

Thus the $i$-th entry of $\beta g$ is $\beta_{1} h_{1}$ and so the $i$-th entry of $\beta g$ is contained in $\beta_{1} K_{\alpha_{1}}$. However, the $i$-th entry of $\beta g$ is also contained in $\beta_{i} K_{\alpha_{1}}$ which gives that $\beta_{i} K_{\alpha_{1}}=\beta_{1} K_{\alpha_{1}}$. Thus there is an element $h=\left(h_{1}, \ldots, h_{\ell}\right) \in\left(K_{\alpha_{1}}\right)^{\ell}$ such that $\beta h=\left(\beta_{1}, \ldots, \beta_{1}\right)$. Clearly, $G^{h} \leqslant$ Aut $(\Gamma h)$; further $G^{h} \leqslant(K \operatorname{wr}(G \pi))^{h}=K \operatorname{wr}(G \pi)$ since $h \in K^{\ell}$. qed

If $\Gamma_{1}$ and $\Gamma_{2}$ are graphs, then the direct product of $\Gamma_{1} \times \Gamma_{2}$ is defined as the graph whose vertex set is the cartesian product $\mathrm{V}\left(\Gamma_{1}\right) \times \mathrm{V}\left(\Gamma_{2}\right)$ and adjacency is defined by the rule that $\left(\gamma_{1}, \gamma_{2}\right),\left(\gamma_{1}^{\prime}, \gamma_{2}^{\prime}\right)$ are adjacent in $\Gamma_{1} \times \Gamma_{2}$ if and only if $\gamma_{1}$ is adjacent to $\gamma_{1}^{\prime}$ in $\Gamma_{1}$ and $\gamma_{2}$ is adjacent to $\gamma_{2}^{\prime}$ in $\Gamma_{2}$. The definition of direct product can be extended to an arbitrary finite number of factors, and in particular, if $\Gamma_{1}$ is a graph and $l \geqslant 2$, then one 
can define $\left(\Gamma_{1}\right)^{l}$ as the graph with vertex set $\mathrm{V}\left(\Gamma_{1}\right)^{l}$ such that $\left(\gamma_{1}, \ldots, \gamma_{l}\right)$ is adjacent to $\left(\gamma_{1}^{\prime}, \ldots, \gamma_{l}^{\prime}\right)$ in $\left(\Gamma_{1}\right)^{l}$ if and only if $\gamma_{i}$ is adjacent to $\gamma_{i}^{\prime}$ in $\Gamma_{1}$ for all $i$. Note that if $\alpha_{1} \in \Gamma_{1}$ and $\alpha=\left(\alpha_{1}, \ldots, \alpha_{1}\right)$ then the neighborhood $\left(\Gamma_{1}\right)^{l}(\alpha)$ of $\alpha$ in $\left(\Gamma_{1}\right)^{l}$ is equal to the cartesian power $\left(\Gamma_{1}\left(\alpha_{1}\right)\right)^{l}$ of the neighborhood of $\alpha_{1}$ in $\Gamma_{1}$. See [K00 for more background on products of graphs and their properties.

Proposition 3.4. Suppose that Hypothesis 3.1 holds, that $\Gamma$ is $M$-arc-transitive and that the inclusion of $G$ into $W$ is normal. Then $\Gamma \cong\left(\Gamma_{1}\right)^{\ell}$ where $\Gamma_{1}$ is the graph whose vertex set is $\Delta$ and edge set is the $M^{(1)}$-orbit of $\left\{\alpha_{1}, \beta_{1}\right\}$ where $\alpha_{1}, \beta_{1} \in \Delta$ are chosen so that $\left(\alpha_{1}, \ldots, \alpha_{1}\right)$ and $\left(\beta_{1}, \beta_{2}, \ldots, \beta_{\ell}\right)$ are connected in $\Gamma$. In particular, $\Gamma$ is not $(G, 2)$-arctransitive.

Proof. Since the inclusion of $G$ into $W$ is normal, we find that $G \pi$ is transitive (Proposition 2.5), and hence we may assume without loss of generality by Theorem 2.3 that $G \leqslant G^{(1)}$ wr $(G \pi)$ and, by Lemma 3.3, that there are $\alpha_{1}, \beta_{1} \in \Delta$ such that $\alpha=\left(\alpha_{1}, \ldots, \alpha_{1}\right)$ and $\beta=\left(\beta_{1}, \ldots, \beta_{1}\right)$ are adjacent in $\Gamma$. Since $M$ is arc-transitive, we find that $M_{\alpha}$ is transitive in $\Gamma(\alpha)$. Further, by Proposition [2.5, $M_{\alpha}=\prod_{j}\left(M^{(j)}\right)_{\alpha_{1}}$. As $\Gamma$ is $M$-arctransitive, $M$ is not regular on $\Omega$. Thus $M^{(j)}$ is the unique minimal normal subgroup of $G^{(j)}$. Since $G^{(j)}=G^{(1)}$, we have that $M^{(j)}=M^{(1)}$ for all $j$. Thus

$$
\Gamma(\alpha)=\prod_{i=1}^{\ell} \beta_{1}\left(M^{(1)}\right)_{\alpha_{1}} .
$$

If $\Gamma_{1}$ denotes the graph whose vertex set is $\Delta$ and whose edge set is the $M^{(1)}$-orbit containing $\left\{\alpha_{1}, \beta_{1}\right\}$ then, by, the remark before the proposition, we have that the neighborhoods of $\alpha$ in $\Gamma$ and in $\left(\Gamma_{1}\right)^{\ell}$ are equal. Since $M$ acts vertex transitively on both $\Gamma$ and $\left(\Gamma_{1}\right)^{\ell}$, we find that $\Gamma=\left(\Gamma_{1}\right)^{\ell}$. As the valency of $\Gamma$ is at least 2 , we obtain that $\Gamma_{1}$ also has valency at least 2 . Hence if $\beta_{2} \in \beta_{1}\left(M^{(1)}\right)_{\alpha_{1}} \backslash\left\{\beta_{1}\right\}$, then both $\left(\beta_{1}, \ldots, \beta_{1}, \beta_{2}\right)$ and $\left(\beta_{1}, \ldots, \beta_{1}, \beta_{2}, \beta_{2}\right)$ are in $\Gamma(\alpha) \backslash\{\beta\}$, however no element of $K_{\alpha_{1}, \beta_{1}}$ wr $\mathrm{S}_{\ell}$ can map one of these points to the other. Hence $G_{\alpha}$ is not 2-transitive on $\Gamma(\alpha)$ and so $\Gamma$ is not $(G, 2)$-arc-transitive. qed

Corollary 3.5. Suppose that Hypothesis 3.1 holds, that $M$ is not regular, and that the inclusion $G \leqslant W$ is normal. Then $\Gamma$ is not $(G, 2)$-arc-transitive.

Proof. Let $\alpha$ be a vertex of $\Gamma$. If $\Gamma$ is $(G, 2)$-arc-transitive, then $G_{\alpha}$ is 2 -transitive on $\Gamma(\alpha)$ and hence $\Gamma$ is $M$-arc-transitive; by Proposition 3.4, this is a contradiction. Thus $\Gamma$ is not $(G, 2)$-arc-transitive. qed

We note that if $G$ is a quasiprimitive group of type $C D$, then its unique minimal normal subgroup $M$ is not regular and $M_{\omega}$ is an $\mathcal{S}$-subgroup with respect to the direct product decomposition of $M$ determined by the simple factors of $M_{\omega}$. Hence By Corollary [3.5, for such a $G$, there exist no $(G, 2)$-arc-transitive graphs. This argument gives an alternative proof of [Pra92, Lemma 5.3(i)].

Finally in this section we exclude the case of abelian $M$. 
Proposition 3.6. Under Hypothesis 3.1, if $\Gamma$ is $(G, 2)$-arc-transitive, then $M$ is nonabelian.

Proof. Suppose that $M$ is abelian. Then $G$ is an affine primitive permutation group and, for $\alpha \in \Omega, G=M \rtimes G_{\alpha}$ where $M$ is viewed as a $k$-dimensional vector space over $\mathbb{F}_{p}$ (see [BP04, Section 3]). Hence in this proof we use additive notation in $M$. Further, $M$, viewed as an $G_{\alpha}$-module, is irreducible. By Lemma 2.2, $M \leqslant(\operatorname{Sym} \Delta)^{\ell}$ and so $M \leqslant M^{(1)} \oplus \cdots \oplus M^{(\ell)}$. As each component $M^{(j)}$ is transitive, and hence regular, on $\Delta$, we find that $\left|M^{(j)}\right|=|\Delta|$. Since $|M|=|\Omega|=|\Delta|^{\ell}=\prod_{j}\left|M^{(j)}\right|$, we obtain that $M=M^{(1)} \oplus \cdots \oplus M^{(\ell)}$ and the components $M^{(j)}$ are permuted transitively by $G_{\alpha}$. In other words, $G_{\alpha}$ preserves a direct sum decomposition of $M$ into $\ell$ components; that is, $G_{\alpha}$ is primitive as a linear group acting on $M$.

The class of $(G, 2)$-arc-transitive graphs with primitive affine groups $G$ were classified by [IP93. We need to consider those lines in [IP93, Table 1] in which the corresponding group is primitive; that is, the entry in the "Prim" column is "P". We will show for these lines that a point stabilizer $G_{\alpha}$ does not preserve a non-trivial direct sum decomposition of $M$.

In Lines $1-2, G_{\alpha}$ is $\mathrm{GL}(k, 2)$ or $\mathrm{S}_{k+1}$ and these groups do not preserve non-trivial direct sum decompositions. In Line $7, G_{\alpha}=\operatorname{P\Gamma L}\left(m, 2^{a}\right)$ acting in dimension $k=m^{a}$ with $m \geqslant 3$ and $a \geqslant 1$. By the discussion in [IP93, (1.3)], $M$ is an irreducible module for $\operatorname{PSL}\left(m, 2^{a}\right)$. We claim that $M$ is a primitive $\operatorname{PSL}\left(m, 2^{a}\right)$-module. If $M$ were imprimitive, then $\operatorname{PSL}\left(m, 2^{a}\right)$ would permute non-trivially an imprimitivity decomposition $M=V_{1} \oplus \cdots \oplus V_{\ell}$ with $\ell \leqslant m^{a}$. On the other hand, if $\left(m, 2^{a}\right) \neq(4,2)$, the minimal degree permutation representation of $\operatorname{PSL}\left(m, 2^{a}\right)$ is on the 1-spaces of the natural module and has degree $\left(2^{m a}-1\right) /\left(2^{a}-1\right)>2^{(m-1) a}$, while the minimal degree of a permutation representation of $\mathrm{SL}(4,2)$ is 8 ; see [Coo78, Table 1]. As $m \geqslant 3$, we have that $m<2^{m-1}$, and so $m^{a}<2^{(m-1) a}$. Further, $4^{1}<8$, and so we obtain that $\operatorname{PSL}\left(m, 2^{a}\right)$ cannot be imprimitive in these cases.

We have, in the remaining two lines, that $G_{\alpha} \cong M_{23}$ or $G_{\alpha} \cong M_{22} .2$; these lines can be be handled similarly. If the underlying module of $M_{23}$ were imprimitive, then $M_{23}$ would be acting on 11 points transitively, which is impossible. If the underlying module for $M_{22} .2$ were imprimitive, then either $M_{22}$ would be acting in dimension less then 10 or it would be acting transitively on at most 10 points; both of these possibilities are clearly impossible by $\left[\mathrm{CCN}^{+} 85\right]$. qed

\section{Simple PLinth}

In this section we will consider the situation described in Hypothesis 3.1 under the additional condition that $M=T$ is a non-abelian simple group. Unlike in the other cases, here we find two examples. Further, we show that these examples are the only possibilities. 
Theorem 4.1. Suppose that Hypothesis 3.1 holds. Assume, further that $M=T$ is a non-abelian simple group and that $\Gamma$ is $(G, 2)$-arc-transitive. Then one of the following is valid.

(1) $T=\mathrm{A}_{6}, \mid$ Aut $_{6}: G \mid \in\{1,2\}, G \neq \mathrm{PGL}(2,9)$, and $|\mathrm{V}(\Gamma)|=36, \Gamma$ is Sylvester's Double Six Graph of valency 5.

(2) $T=\operatorname{Sp}(4,4), G=$ Aut $\mathrm{Sp}(4,4),|\mathrm{V}(\Gamma)|=14,400=120^{2}$, and $\Gamma$ is a graph of valency 17.

In both cases, $G$ is quasiprimitive.

Proof. Let $\alpha \in \mathrm{V}(\Gamma)$. By [BPS04, Theorem 6.1], $T_{\alpha}$ is non-trivial. As $\Gamma$ is connected and $G_{\alpha}$ is 2-transitive, and in particular quasiprimitive, on $\Gamma(\alpha)$, Lemma 3.2 gives that $\Gamma$ is $T$-arc-transitive. In particular $\Gamma(\alpha)$ is a $T_{\alpha}$-orbit. As $T$ is simple, the possibilities for $T$ and $T_{\alpha}$ are given in [BPS04, Theorem 6.1]. In particular, $T$ is isomorphic to one of the groups $\mathrm{A}_{6}, \mathrm{M}_{12}, \mathrm{Sp}\left(4,2^{a}\right)$, with $a \geqslant 2$, or $\mathrm{P} \Omega^{+}(8, q)$. We analyze each of these cases below.

$T=\mathrm{A}_{6},|\mathrm{~V}(\Gamma)|=36$ : In this case calculation with GAP [GAP13] or Magma [BCP97] can show that $G$ has a suborbit of size 5 on which $G_{\alpha}$ acts 2 -transitively if and only if $\mathrm{S}_{6} \leqslant G$. The corresponding 2-arc-transitive graph is undirected, connected and is of valency 5 as described in item (1).

$T=\mathrm{M}_{12}$ and $|\mathrm{V}(\Gamma)|=144$ : Calculation with GAP GAP13] or Magma [BCP97] shows that $T$ has no suborbit on which $G_{\alpha}$ is 2-transitive and the corresponding graph is connected. Hence no graph arises in this case.

$T=\operatorname{Sp}(4, q)$ with $q=2^{a}$ and $a \geqslant 2$ : The group $G$ is contained in Aut $T \cong \operatorname{Sp}(4, q) \cdot C_{a} . C_{2}$ where $C_{a}$ is the group of field automorphisms and $C_{2}$ is generated by a graph automorphism. Further, $T_{\alpha}=Z\langle\sigma\rangle$ where $Z$ is a subgroup of order $q^{2}+1$ of a Singer cycle and $\sigma$ is an element of order 4; see [BPS04, Lemma 5.2]. Let $\gamma \in \Gamma(\alpha)$ and let $x \in T$ such that $\gamma=\alpha x$.

We claim that $Z \cap Z^{x}=1$. Suppose that $1 \neq Z_{1}=Z \cap Z^{x}$. Then $Z_{1}$ is cyclic of odd order and suppose that $R$ is a cyclic subgroup of $Z_{1}$ with odd prime order $r$. Then $r \mid q^{2}+1$, and so $r \nmid q^{2}-1$, and hence $r \nmid q-1$ and $r \nmid q^{3}-1=(q-1)\left(q^{2}+q+1\right)$. Thus $r$ is a primitive prime divisor of $q^{4}-1$ and hence the centralizer of $R$ in $\operatorname{GL}(4, q)$ is a full Singer cycle $C_{q^{4}-1}$ Hup67, 7.3 Satz]. Hence $Z$ and $Z^{x}$ are subgroups of the same $C_{q^{4}-1}$, which implies that $Z=Z^{x}$. Since $T_{\alpha}=\mathrm{N}_{T}(Z)$ and $T_{\gamma}=\mathrm{N}_{T}\left(Z^{x}\right)$ we find that $T_{\alpha}=T_{\gamma}$. As $T_{\alpha}$ is self-normalizing in $T$, we obtain that $\alpha=\gamma$ which is a contradiction as $\gamma \in \Gamma(\alpha)$ is assumed. This shows that $Z \cap Z^{x}=1$ as claimed.

As $Z \cap Z^{x}=1, Z$ is faithful on $\Gamma(\alpha)$. As $Z$ is normal in $G_{\alpha}$ and $G_{\alpha}$ is 2-transitive on $\Gamma(\alpha), Z$ is transitive, and hence regular, on $\Gamma(\alpha)$. Thus $|\Gamma(\alpha)|=q^{2}+1$. As $\left|G_{\alpha}\right| \leqslant 8 a\left(q^{2}+1\right)$, and $G_{\alpha}$ is 2 -transitive on $\Gamma(\alpha)$, it follows that $a=2$. If $a=2$, then there exists a corresponding 2-arc-transitive graph $\Gamma$, which is given in item (2).

$T=\mathrm{P} \Omega^{+}(8, q)$ : In this case $T_{\alpha}=G_{2}(q)$. If $q \geqslant 3$, then $G_{2}(q)$ does not have a 2-transitive permutation representation. As is well-known, $G_{2}(2) \cong \operatorname{PSU}(3,3) .2$, and $\operatorname{PSU}(3,3) .2$ has a 2 -transitive representation of degree $q^{3}+1=28$. However, computation 
with GAP GAP13] or Magma BCP97] shows that $T_{\alpha}$ has no suborbit of size 28, and hence this case does not arise. qed

Computation with GAP [GAP13] or Magma [BCP97] can verify that the 2-arc-transitive graphs with $T=\mathrm{A}_{6}$ and $T=\mathrm{Sp}(4,4)$ do in fact exist. The graph with $T=\mathrm{A}_{6}$ is also known as Sylvester's Double Six Graph, it has 36 vertices and valency 5 (see BCN89, 13.1.2 Theorem]). Its full automorphism group is Aut $A_{6} \cong \operatorname{PLL}(2,9)$. The graph with $T=\operatorname{Sp}(4,4)$ has $120^{2}=14,400$ vertices and valency 17 . Both graphs that appear in this context can be described using the generalized quadrangle associated with the nondegenerate alternating form stabilized by $\mathrm{Sp}(4, q)$, but giving such a description is beyond the scope of the present paper.

\section{NON-SIMPLE PLINTH}

We continue working under Hypothesis 3.1. We know from Proposition 3.6 that if $\Gamma$ is $(G, 2)$-arc-transitive, then $M$ is non-abelian. Further, the situation when $\Gamma$ is $(G, 2)$-arctransitive and $M$ is simple is fully described in Section 4. Hence from now of we will focus on the case when $M$ is non-abelian and non-simple. We will also assume that $M$ is not regular, as for the case when $M$ is regular, the graph will be a Cayley graph; see [Bad93.

We note that, using [LSS15, Theorem 1.3], our analysis could by somewhat simplified if we assumed that $G$ was quasiprimitive. However, we opt for keeping our general conditions set up in Hypothesis 3.1 .

Theorem 5.1. Suppose that Hypothesis 3.1 is valid, that $\Gamma$ is $(G, 2)$-arc-transitive and that $M$ is non-regular and non-simple. Then $G \pi$ is transitive and the inclusion $G \leqslant W$ is of type $\mathrm{CD}_{2 \nsim}$.

Proof. By Corollary 3.5, the inclusion $G \leqslant W$ is not normal. We need to show that the cases when $G \pi$ is intransitive, or the inclusion $G \leqslant W$ has type $\mathrm{CD}_{1 S}, \mathrm{CD}_{2 \sim}$, or $\mathrm{CD}_{3}$ are impossible. Assume, by contradiction, that one of these cases is valid and Theorem 2.9 applies. Let $\alpha$ be a vertex of $\Gamma$. Whenever, in Theorem 2.9 , the stabilizer $M_{\alpha}$ is an $\mathcal{S}$-subgroup with $k_{1} \geqslant 2$, there exists, by Theorem 2.6, a normal embedding $G \leqslant \operatorname{Sym} \Xi \mathrm{wr}_{k_{1}}$. If $k_{1}=k$, then $k_{1} \geqslant 2$ by the conditions of the theorem. If the inclusion type is $\mathrm{CD}_{1 S}$, then, by the definition of this inclusion type, a component $M^{(1)}$ contains a full diagonal subgroup in $T_{i_{1}} \times T_{i_{2}}$, but there is also a simple factor $T$ of $M$ such that the projection $\left(M^{(1)}\right)_{\delta} \bar{T} / \bar{T}$ is a proper subgroup of $T$. Hence this $T$ is different from $T_{i_{1}}$ and $T_{i_{2}}$ and this implies that $k_{1} \geqslant 2$ also in this case. Hence in these cases, we obtain the desired contradiction by Corollary 3.5. Thus we are left with the possibilities of Theorem 2.9(1)-(4) in which $M_{\alpha}$ is not an $\mathcal{S}$-subgroup. In these cases $T \cong \operatorname{Sp}(n, q)$ where the possibilities for the inclusion type, $n, q$, and $k_{1}$ are summarized in Table 2 .

In each of the cases in Table 2, we have that

$$
\left(\mathrm{D}_{q+1}\right)^{k_{1}} \leqslant M_{\alpha} \leqslant\left(\mathrm{D}_{q+1} \cdot 2\right)^{k_{1}} .
$$




\begin{tabular}{|c|c|c|c|}
\hline $\begin{array}{c}\text { inclusion } \\
\text { type }\end{array}$ & $n$ & $q$ & $k_{1}$ \\
\hline intransitive & 4 & $2^{a} \geqslant 4$ & $k$ \\
\hline $\mathrm{CD}_{2 \sim}$ & 4 & $2^{a} \geqslant 4$ & $k$ \\
\hline $\mathrm{CD}_{3}$ & 6 & 2 & $k \geqslant 3$ \\
\hline $\mathrm{CD}_{1 S}$ & 4 & $2^{a} \geqslant 4$ & $k / 2 \geqslant 2$ \\
\hline
\end{tabular}

TABLE 2 .

Let $A$ and $B$ denote the subgroups of the left and the right hand side, respectively, of (3). Hence in each case we have that $A \leqslant M_{\alpha} \leqslant B$. Let $C$ denote the common Hall 2 '-subgroup of $A$ and $B$. Thus $C$ is isomorphic to $\left(C_{q+1}\right)^{k_{1}}$. By the conditions, $C$ is a subgroup of $M_{\alpha}$ and $B=\left(\mathrm{D}_{q+1} \cdot 2\right)^{k_{1}}$. For $i=1, \ldots, k_{1}$, we let $\sigma_{i}$ denote the $i$-th coordinate projection $B \rightarrow \mathrm{D}_{q+1} \cdot 2$.

Claim 1. If $N$ is a normal subgroup of $G_{\alpha}$ contained in $C$, then $N=\left(C_{s}\right)^{k_{1}}$ where $s$ is a divisor of $q+1$. Conversely, each such subgroup of $C$ is normal in $G_{\alpha}$.

Proof. We have that $N \sigma_{i} \leqslant C_{q+1}$. The subgroup $C$, being characteristic in $M_{\alpha}$, is normalized by $G_{\alpha}$, and $G_{\alpha}$ preserves the direct decomposition $C=\left(C_{q+1}\right)^{k_{1}}$. Thus $N \sigma_{i}$ is independent of $i$. Therefore $N \sigma_{i}$ is a cyclic subgroup of order $s$, say, of $C_{q+1}$, and hence $N$ is a subdirect subgroup of $\left(C_{s}\right)^{k_{1}}$. Suppose that $x_{1} \in C_{s}$. Then there is an element of the form $\left(x_{1}, \ldots, x_{k_{1}}\right)$ in $N$ with $x_{i} \in C_{s}$. Suppose that $x \in \mathrm{D}_{q+1}$ is an involution that conjugates each element of $C_{q+1}$ to its inverse. Since $M_{\alpha}$ contains $\left(\mathrm{D}_{q+1}\right)^{k_{1}}$, $(1, x, \ldots, x) \in M_{\alpha}$, and hence

$$
\left(x_{1}, x_{2}, \ldots, x_{k_{1}}\right)^{(1, x, \ldots, x)}=\left(x_{1}, x_{2}^{-1}, \ldots, x_{k_{1}}^{-1}\right) \in N,
$$

which implies that

$$
\left(x_{1}, x_{2}, \ldots, x_{k_{1}}\right)\left(x_{1}, x_{2}^{-1}, \ldots, x_{k_{1}}^{-1}\right)=\left(x_{1}^{2}, 1, \ldots, 1\right) \in N .
$$

Since $s$ is odd, the order $\left|x_{1}\right|$ of $x_{1}$ is coprime to 2 , which gives that $\left(x_{1}, 1, \ldots, 1\right) \in N$. This shows that $N$ contains $N \sigma_{1}$. The same argument shows that $N$ contains $N \sigma_{i}$ for all $i$, and hence $N=\left(C_{s}\right)^{k_{1}}$ as claimed.

A subgroup of the form $\left(C_{s}\right)^{k_{1}}$ is normalized by $G_{\alpha}$, since $\left(C_{q+1}\right)^{k_{1}}$ is normalized by $G_{\alpha}$ (it is the unique $2^{\prime}$-subgroup of $\left.M_{\alpha}\right)$ and $\left(C_{s}\right)^{k_{1}}$ is the largest subgroup of $\left(C_{q+1}\right)^{k_{1}}$ with exponent $s$, and hence it is characteristic in $\left(C_{q+1}\right)^{k_{1}}$ and normal in $G_{\alpha}$. qed

Suppose that $\Gamma$ is a $(G, 2)$-arc-transitive graph, as assumed in the theorem. We know that $Q=G_{\alpha}^{\Gamma(\alpha)}$ is a 2-transitive group. In particular $G_{\alpha}$ is primitive on $\Gamma(\alpha)$ and so $M_{\alpha}$ is either transitive on $\Gamma(\alpha)$ or trivial. By Lemma 3.2, we obtain that $M_{\alpha}$ is transitive. Since $M_{\alpha} \unlhd G_{\alpha}$ and $M_{\alpha}$ is solvable, we find that $Q$ is an affine 2-transitive group. In particular the socle $V$ of $Q$ is $V=\mathbb{Z}_{p}^{d}$ with some prime $p$ and $Q=V \rtimes Q_{\beta}=\mathbb{Z}_{p}^{d} \rtimes G_{\alpha, \beta}^{\Gamma(\alpha)}$. 
A linear group $H \leqslant \mathrm{GL}(d, p)$ is said to be monomial if $H$ preserves a direct sum decomposition $V_{1} \oplus \cdots \oplus V_{d}$ of $V=\left(\mathbb{F}_{p}\right)^{d}$ with subspaces of dimension 1. If $H$ is monomial, then $H$ cannot be transitive on $V \backslash\{0\}$, and hence $V \rtimes H$ cannot be 2-transitive on $V$. In particular, the linear group $Q_{\beta}$ above is not monomial.

Set $G_{\alpha}^{[1]}$ to be the kernel of $G_{\alpha}$ in its action on $\Gamma(\alpha)$. Define $M_{\alpha}^{[1]}$ accordingly and note that $M_{\alpha}^{[1]}$ is normal in $G_{\alpha}$. Recall that $C=\left(C_{q+1}\right)^{k_{1}}$ as defined above and that $C$ is normal in $G_{\alpha}$.

Claim 2. $C \leqslant M_{\alpha}^{[1]}$.

Proof. Let $C_{1}=C \cap M_{\alpha}^{[1]}=C \cap G_{\alpha}^{[1]}$. Then $C_{1}$ is normal in $G_{\alpha}$. Hence $C_{1}=\left(C_{s}\right)^{k_{1}}$ with some $s \mid q+1$ by Claim 1. Thus $C / C_{1}=C /\left(C \cap G_{\alpha}^{[1]}\right)=C G_{\alpha}^{[1]} / G_{\alpha}^{[1]}$ can be considered as a normal subgroup of $G_{\alpha}^{\Gamma(\alpha)}$. If $C_{1} \neq C$, then this is a non-trivial normal subgroup of $G_{\alpha}^{\Gamma(\alpha)}$ and hence it contains a minimal normal subgroup of the form $V=\left(C_{r}\right)^{k_{1}}$ with some prime divisor $r$ of $q+1$. On the other hand, since $G_{\alpha}$ permutes the $k_{1}$ factors of $C$, $G_{\alpha}$ induces a monomial subgroup on $\left(C_{r}\right)^{k}$, and hence $G_{\alpha}$ cannot be transitive on the set of non-trivial elements of $V$ (see the remark above). This is a contradiction, and hence $C_{1}=C$, which means that $C \leqslant M_{\alpha}^{[1]}$. qed

Let $\beta \in \Gamma(\alpha)$. By Claim 2, $C \leqslant M_{\beta}$. Since $M_{\beta}^{[1]}$ is $G$-conjugate to $M_{\alpha}^{[1]}$, it contains the unique Hall $2^{\prime}$-subgroup of $M_{\beta}$. On the other hand, this Hall $2^{\prime}$-subgroup is equal to $C$, and hence $C \leqslant M_{\beta}^{[1]}$, and so $C \leqslant M_{\delta}$ for all $\delta \in \Gamma(\beta)$. Then the same argument implies that if $\delta \in \Gamma(\beta)$, then $C \leqslant M_{\delta}^{[1]}$. Since $\Gamma$ is assumed to be connected, this implies that $C$ acts trivially on the vertex set $\Omega$ of $\Gamma$, which is impossible, as $G$ is assumed to be a subgroup of Sym $\Omega$.

This shows that the assumption that either $G \pi$ is intransitive or that the inclusion $G \leqslant W$ is one of the types $\mathrm{CD}_{1 S}, \mathrm{CD}_{2 \sim}$, or $\mathrm{CD}_{3}$ always leads to a contradiction. Hence the inclusion $G \leqslant W$ has to have type $\mathrm{CD}_{2 \nsim}$. qed

Now Theorem 1.1 is a consequence of Proposition 3.6, Corollary 3.5, Theorem 4.1 and Theorem 5.1.

\section{The Li-Seress EXAMPles}

By Theorem 1.1, if $\Gamma$ is a connected $(G, 2)$-arc-transitive graph for an innately transitive permutation group $G$ with a non-regular, non-simple plinth, then an inclusion $G \leqslant W$ into a wreath product $W$ in product action as in Hypothesis 2.1 has to have type $\mathrm{CD}_{2 \chi}$. On the other hand, we do not know if such $(G, 2)$-arc-transitive graphs exist. The information about the finite simple factor $T$ of the plinth and its factorization given in Lemma 2.4 is not as strong in this case as in the cases described by Theorem 2.9. Thus deciding if such examples exist seems to be a difficult problem. By Theorem 1.1, the O'Nan-Scott type of a quasiprimitive group $G$ in an example must be PA. We show in this section that the 


\begin{tabular}{|c|c|c|c|c|c|c|c|c|c|c|}
\hline$q$ & 5 & 7 & 9 & 9 & 9 & 11 & 19 & 23 & 29 & 59 \\
\hline$A$ & $P_{1}$ & $P_{1}$ & $\mathrm{~S}_{4}$ & $P_{1}$ & $\mathrm{~A}_{5}$ & $P_{1}$ & $P_{1}$ & $P_{1}$ & $P_{1}$ & $P_{1}$ \\
\hline$B$ & $\mathrm{~A}_{4}$ & $\mathrm{~S}_{4}$ & $\mathrm{~A}_{5}$ & $\mathrm{~A}_{5}$ & $\mathrm{~A}_{5}$ & $\mathrm{~A}_{5}$ & $\mathrm{~A}_{5}$ & $\mathrm{~S}_{4}$ & $\mathrm{~A}_{5}$ & $\mathrm{~A}_{5}$ \\
\hline$A \cap B$ & $C_{2}$ & $C_{3}$ & $C_{2} \times C_{2}$ & $\mathrm{~S}_{3}$ & $\mathrm{D}_{5}$ & $C_{5}$ & $C_{3}$ & 1 & $C_{2}$ & 1 \\
\hline
\end{tabular}

TABLE 3. Exceptional factorizations of $\operatorname{PSL}(2, q)$

examples of $(G, 2)$-arc-transitive graphs with quasiprimitive groups of type PA in LS06] do not admit an inclusion $G \leqslant W$ of type $\mathrm{CD}_{2 \not}$.

In all the examples of $(G, 2)$-arc-transitive graphs in [LS06], $G$ is a quasiprimitive group with socle $M=T^{k}$ where $T \cong \operatorname{PSL}(2, q)$ with some $q$. Thus to rule out inclusions of type $\mathrm{CD}_{2 \nsim}$ for these groups, we need to review some known facts about factorizations of $\operatorname{PSL}(2, q)$. The following lemma is a consequence of [LPS90, Tables 1 and 3] and Dickson's description of the maximal subgroups of $\operatorname{PSL}(2, q)$ (see Giu07] for an easy reference).

Lemma 6.1. Suppose that $T=\operatorname{PSL}(2, q)$ with some prime power $q \geqslant 4$.

(1) If $q \equiv 1(\bmod 4)$ and $q \notin\{5,9,29\}$, then $T$ does not have a factorization $T=A B$ with proper subgroups $A, B<T$.

(2) If $q \not \equiv 1(\bmod 4)$, then $T$ can be factorized as $T=A B$, where $A$ is a maximal parabolic subgroup and

$$
B= \begin{cases}\mathrm{D}_{(q+1)} & \text { when } q \text { is even } \\ \mathrm{D}_{(q+1) / 2} & \text { when } q \text { is odd. }\end{cases}
$$

Further,

$$
|A \cap B|=\left\{\begin{array}{l}
2 \text { when } q \text { is even; } \\
1 \text { when } q \text { is odd. }
\end{array}\right.
$$

(3) If $q \not \equiv 1(\bmod 4)$ and $q \notin\{7,11,19,23,59\}$, then the only factorization $T=A B$ of $T$ with maximal subgroups $A, B$ is as in (2).

(4) If $q \in\{5,7,9,11,19,23,29,59\}$, then the factorizations $\operatorname{PSL}(2, q)=A B$ with maximal subgroups $A$ and $B$ are either listed in part (2) or in Table 3, where $P_{1}$ denotes a maximal parabolic subgroup in $T$.

The information about the factorizations of $\operatorname{PSL}(2, q)$ in Lemma 6.1 allows us to characterize inclusions $G \leqslant W$ in wreath products in product action of innately transitive groups $G$ whose plinths are isomorphic to $\operatorname{PSL}(2, q)^{k}$.

Theorem 6.2. Suppose that $G$ is an innately transitive group on $\Omega$ whose plinth $M$ is isomorphic to $\operatorname{PSL}(2, q)^{k}$ with some prime power $q \geqslant 4$ and $k \geqslant 1$. Let $T$ be a simple factor of $M$, set $\bar{T}=\mathrm{C}_{M}(T)$ and let $\omega \in \Omega$.

(1) If $q \equiv 1(\bmod 4)$ and $q \notin\{5,9,29\}$, then every inclusion of $G \leqslant W$ satisfying Hypothesis 2.1 is normal. 
(2) Suppose $q \equiv 3(\bmod 4)$ and $q \notin\{7,11,19\}$ and assume that $G$ admits a nonnormal inclusion $G \leqslant W$ satisfying Hypothesis 2.1. Then $M$ is regular and $G$ admits a normal inclusion $G \leqslant \operatorname{Sym} \Xi \mathrm{wr}_{k}$ where $|\Xi|=|T|$.

(3) If $q$ is even and $G \leqslant W$ is a non-normal inclusion such that Hypothesis 2.1 holds, then $M_{\omega} \bar{T} / \bar{T} \leqslant C_{2}$.

(4) If $q \in\{5,7,9,11,19,29\}$ and $G \leqslant W$ is a non-normal inclusion satisfying Hypothesis [2.1, then either $M_{\omega} \bar{T} / \bar{T}=1$ and $M$ is regular or $M_{\omega} \bar{T} / \bar{T}$ is a non-trivial subgroup of the $A \cap B$ column of a corresponding column of Table 3 .

Proof. Suppose that $G$ is an innately transitive group as in the conditions of the theorem and that $G \leqslant W$ is an inclusion satisfying Hypothesis 2.1. Let $\pi: W \rightarrow \mathrm{S}_{\ell}$ be the natural projection. By Theorem 2.9 (1), either $G \pi$ is transitive, or $T \cong \operatorname{PSL}(2,9) \cong \mathrm{A}_{6}$. Further, if $G \pi$ is transitive, but the inclusion is not normal, then, by Theorem 2.9)(2)-(3), either $T \cong \operatorname{PSL}(2,9) \cong \mathrm{A}_{6}$, or the inclusion type is $\mathrm{CD}_{2 \chi}$.

Let first consider the case when the inclusion type is not $C_{2}$. Hence $T \cong P S L(2,9) \cong A_{6}$ and either $G \pi$ is intransitive, or the inclusion is one of the types $\mathrm{CD}_{2} \sim$ or $\mathrm{CD}_{1 S}$. Then, by Theorem $2.9(2), M_{\omega} \bar{T} / \bar{T} \cong \mathrm{D}_{5}$. Since $\mathrm{D}_{5}$ appears in the $A \cap B$ column of Table 3 , the result holds in this case.

Let us consider now the case of $\mathrm{CD}_{2 \chi}$ inclusions. In this case, $T$ is contained in exactly two components $M^{\left(j_{1}\right)}$ and $M^{\left(j_{2}\right)}$. Let $\delta \in \Delta$ and set $\omega=(\delta, \ldots, \delta)$. Then, by Lemma 2.4, $A=\left(M^{\left(j_{1}\right)}\right)_{\delta} \bar{T} / \bar{T}$ and $B=\left(M^{\left(j_{2}\right)}\right)_{\delta} \bar{T} / \bar{T}$ are proper subgroups of $T$ satisfying $T=A B$ and $M_{\omega} \bar{T} / \bar{T} \leqslant A \cap B$. If $q \equiv 1(\bmod 4)$ and $q \notin\{5,9,29\}$, then, by Lemma $6.1(1)$, $T=\operatorname{PSL}(2, q)$, does not admit a factorization with two proper subgroups, and hence, in this case, inclusions of type $\mathrm{CD}_{2 \nsim}$ are not possible. This proves claim (1). If $q \equiv 3$ $(\bmod 4)$, but $q \notin\{7,11,19\}$ and $T=A B$ with proper subgroups $A$ and $B$ of $T$, then, by Lemma $6.1(3)-(4), A \cap B=1$. Thus, the projection $M_{\omega} \bar{T} / \bar{T}$ is trivial. Since this projection is independent of the choice of $T, M_{\omega}=1$ and hence $M$ is regular. Then $M_{\omega}$ satisfies the conditions in Theorem 2.6 with the direct product decomposition of $M=T^{k}$ into $k$ simple factors, and hence $G$ can be embedded into Sym $\Xi$ wr $S_{k}$ such that $\Xi$ is the right coset space of the trivial subgroup in $T$. This proves $(2)$.

Finally, if $q$ is even or $q \in\{5,7,9,11,19,29\}$ and $G \leqslant W$ is an inclusion of type $C_{2 \chi}$, then $M_{\omega} \bar{T} / \bar{T} \leqslant A \cap B$, where $A, B$ are the subgroups defined above. If $q$ is even, then, by Lemma 6.1(2), $A \cap B \cong C_{2}$, and so (3) follows. If $q$ is one of the odd prime-powers, listed above, then either $A \cap B=1$, and so $M$ is regular, or $A$ and $B$ must occur in Table 3 , and so claim (4) follows also. qed

Corollary 6.3. Suppose that Hypothesis 3.1 holds and that $M$ is non-regular and is isomorphic to $\operatorname{PSL}(2, q)^{k}$ where $k \geqslant 1, q \geqslant 4$ is an odd prime-power and $q \notin\{5,7,9,11,19,29\}$. Then $\Gamma$ is not $(G, 2)$-arc-transitive.

Proof. Suppose that $G, W$ and $\Gamma$ are as in Hypothesis [3.1. If $G \leqslant W$ is a normal inclusion, then $\Gamma$ is not $(G, 2)$-arc-transitive by Corollary 3.5 . Since $T \neq \operatorname{PSL}(2,9) \cong \mathrm{A}_{6}$, Theorem 2.9 implies that $G \pi$ is transitive and the type of the inclusion is $C_{2}$. Thus, 


\begin{tabular}{|c|c|c|}
\hline Example & conditions for $q$ & $M_{\omega} \bar{T} / \bar{T}$ \\
\hline Example 3.2 & $q$ is prime, $q \equiv \pm 1 \quad(\bmod 16)$ & $\mathrm{S}_{4}$ \\
\hline Example 3.3 & any & maximal parabolic \\
\hline Example 3.4 & $q$ is prime, $q \equiv \pm 1 \quad(\bmod 16)$ & $\mathrm{S}_{4}$ \\
\hline Example 4.1 & $q$ is prime, $q \equiv \pm 1 \quad(\bmod 5)$ & $\mathrm{D}_{5}$ \\
\hline Example 5.3 & $q \equiv \pm 1(\bmod 8)$ & $\mathrm{S}_{3}$ \\
\hline
\end{tabular}

TABLE 4. The Li-Seress Examples

by Lemma 2.4, $T=\operatorname{PSL}(2, q)$ must admit a factorization $T=A B$ with proper subgroups $A$ and $B$. Thus the conditions of Lemma 6.1(3) must be valid with $q$ odd. Hence by Theorem $6.2(2)$, there exists a normal inclusion $G \leqslant \operatorname{Sym} \Xi w r S_{k}$. Therefore Proposition 3.4 implies that $\Gamma$ is not $(G, 2)$-arc-transitive. qed

Finally, we show that the Li-Seress examples cannot be embedded into wreath products in product action.

Corollary 6.4. Suppose that $\Gamma$ is one of the $(G, 2)$-arc-transitive graphs in Examples 3.2, 3.3, 3.4, 4.1, or 5.3 in [LS06]. Then $G$ cannot be embedded into a wreath product $W$ as in Hypothesis 2.1.

Proof. Suppose that $\Gamma$ is one of the $(G, 2)$-arc-transitive examples in [LS06] for a quasiprimitive group $G$ of O'Nan-Scott type PA. In each of the cases, soc $G=M=T^{k}$ where $T \cong \operatorname{PSL}(2, q)$ with some prime-power $q$. Let $\omega$ be a vertex of $\Gamma$. The conditions for $q$ and for the projection $M_{\omega} \bar{T} / \bar{T}$ are in Table 4. Suppose that $G \leqslant W$ is an inclusion as in Hypothesis 2.1. By Corollary 6.3, $q \in\{5,7,9,11,19,29\}$. By Theorem 2.9, if $G \pi$ is intransitive or the type of the inclusion is $\mathrm{CD}_{1 S}$ or $\mathrm{CD}_{2 \sim}$, then $T=\mathrm{A}_{6} \cong \operatorname{PSL}(2,9)$ and $M_{\omega} \bar{T} / \bar{T} \cong \mathrm{D}_{5}$. However, this is not possible by Table 4 . Inclusions of type $\mathrm{CD}_{3}$ are not possible by Theorem 2.9 (3). Thus the inclusion $G \leqslant W$ has type $C_{2}$. In this case, Lemma 2.4 implies that $T=\operatorname{PSL}(2, q)$ admits a factorization $T=A B$ with proper subgroups such that $M_{\omega} \bar{T} / \bar{T} \leqslant A \cap B$. Comparing the possibilities in Tables 3 and 4 we find that this is impossible. Hence no inclusion $G \leqslant W$ satisfying Hypothesis 2.1 is possible. qed

\section{REFERENCES}

[Bad93] Robert W. Baddeley. Two-arc transitive graphs and twisted wreath products. J. Algebraic Combin., 2(3):215-237, 1993.

[BCN89] A.E. Brouwer, A.M. Cohen, and A. Neumaier. Distance-regular graphs. Ergebnisse der Mathematik und ihrer Grenzgebiete. Springer, 1989.

[BCP97] Wieb Bosma, John Cannon, and Catherine Playoust. The Magma algebra system I: The user language. J. Symbolic Comput., 24:235-265, 1997. 
[BP98] Robert W. Baddeley and Cheryl E. Praeger. On classifying all full factorisations and multiplefactorisations of the finite almost simple groups. J. Algebra, 204(1):129-187, 1998.

[BP03] Robert W. Baddeley and Cheryl E. Praeger. On primitive overgroups of quasiprimitive permutation groups. J. Algebra, 263(2):294-344, 2003.

[BP04] John Bamberg and Cheryl E. Praeger. Finite permutation groups with a transitive minimal normal subgroup. Proc. Lond. Math. Soc., III. Ser., 89(1):71-103, 2004.

[BPS04] Robert W. Baddeley, Cheryl E. Praeger, and Csaba Schneider. Transitive simple subgroups of wreath products in product action. J. Aust. Math. Soc., 77(1):55-72, 2004.

[BPS06] Robert W. Baddeley, Cheryl E. Praeger, and Csaba Schneider. Innately transitive subgroups of wreath products in product action. Trans. Amer. Math. Soc., 358(4):1619-1641 (electronic), 2006.

[BPS07] Robert W. Baddeley, Cheryl E. Praeger, and Csaba Schneider. Quasiprimitive groups and blow-up decompositions. J. Algebra, 311(1):337-351, 2007.

[BPS08] Robert W. Baddeley, Cheryl E. Praeger, and Csaba Schneider. Intransitive Cartesian decompositions preserved by innately transitive permutation groups. Trans. Amer. Math. Soc., 360(2):743-764 (electronic), 2008.

$\left[\mathrm{CCN}^{+} 85\right]$ J. H. Conway, R. T. Curtis, S. P. Norton, R. A. Parker, and R. A. Wilson. Atlas of finite groups. Oxford University Press, Eynsham, 1985. Maximal subgroups and ordinary characters for simple groups, With computational assistance from J. G. Thackray.

[Coo78] Bruce N. Cooperstein. Minimal degree for a permutation representation of a classical group. Israel J. Math., 30(3):213-235, 1978.

[FP99a] Xin Gui Fang and Cheryl E. Praeger. Finite two-are transitive graphs admitting a ree simple group. Communications in Algebra, 27(8):3755-3769, 1999.

[FP99b] Xin Gui Fang and Cheryl E. Preager. Fintte two-arc transitive graphs admitting a suzuki simple group. Communications in Algebra, 27(8):3727-3754, 1999.

[GAP13] The GAP Group. GAP - Groups, Algorithms, and Programming, Version 4.6.5, 2013.

[Giu07] Michael Giudici. Maximal subgroups of almost simple groups with socle $\operatorname{PSL}(2, q)$. arXiv:math/0703685, 2007.

[HNP99] Akbar Hassani, Luz R. Nochefranca, and Cheryl E. Praeger. Two-arc transitive graphs admitting a two dimensional projective linear group. J. Group Theory, 2(4):335-353, 1999.

[Hup67] B. Huppert. Endliche Gruppen. Number v. 1 in Endliche Gruppen. Springer, 1967.

[IK00] W. Imrich and S. Klavžar. Product graphs, structure and recognition. Wiley-Interscience series in discrete mathematics and optimization. Wiley, 2000.

[IP93] A.A. Ivanov and Cheryl E. Praeger. On finite affine 2-arc transitive graphs. Eur. J. Comb., 14(5):421-444, 1993.

[Kov89] L.G. Kovács. Primitive subgroups of wreath products in product action. Proc. Lond. Math. Soc., III. Ser., 58(2):306-322, 1989.

[LPS90] Martin W. Liebeck, Cheryl E. Praeger, and Jan Saxl. The maximal factorizations of the finite simple groups and their automorphism groups. Mem. Amer. Math. Soc., 86(432):iv+151, 1990.

[LS06] Cai Heng Li and Ákos Seress. Constructions of quasiprimitive two-arc transitive graphs of product action type. In Finite geometries, groups, and computation, pages 115-123. Walter de Gruyter GmbH \& Co. KG, Berlin, 2006.

[LSS15] Cai Heng Li, kos Seress, and Shu Jiao Song. s-arc-transitive graphs and normal subgroups. Journal of Algebra, 421(0):331 - 348, 2015. Special issue in memory of kos Seress.

[MS12] J. Morris and P. Spiga. ^-transitive digraphs preserving a cartesian decomposition. ArXiv e-prints, March 2012.

[Pra90] Cheryl E. Praeger. The inclusion problem for finite primitive permutation groups. Proc. Lond. Math. Soc., III. Ser., 60(1):68-88, 1990. 
[Pra92] Cheryl E. Praeger. An O'Nan-Scott theorem for finite quasiprimitive permutation groups and an application to 2-arc transitive graphs. J. Lond. Math. Soc., II. Ser., 47(2):227-239, 1992.

[PS03] Cheryl E. Praeger and Csaba Schneider. Ordered triple designs and wreath products of groups. In Statistics and science: a Festschrift for Terry Speed, volume 40 of IMS Lecture Notes Monogr. Ser., pages 103-113. Inst. Math. Statist., Beachwood, OH, 2003.

[PS07] Cheryl E. Praeger and Csaba Schneider. Three types of inclusions of innately transitive permutation groups into wreath products in product action. Israel J. Math., 158:65-104, 2007.

[PS12] Cheryl E. Praeger and Csaba Schneider. Embedding permutation groups into wreath products in product action. J. Aust. Math. Soc., 92(1):127-136, 2012.

[Ser07] Ákos Seress. Toward the classification of s-arc transitive graphs. In Proc. Groups St Andrews 2005, volume 340 of London Math. Soc. Lecture Notes Series, pages 401-414, 2007.

[Tut47] W. T. Tutte. A family of cubical graphs. Mathematical Proceedings of the Cambridge Philosophical Society, 43:459-474, 101947.

[Wei81] Richard Weiss. The nonexistence of 8-transitive graphs. Combinatorica, 1(3):309-311, 1981.

(C. H. Li) School of Mathematics and Statistics, The University of Western Australia, 35 Stirling Highway 6009 Crawley, Western Australia, cai.heng.li@uwa.edu.au WWW.MATHS.UWA.EDU.AU/ LI

(C. E. Praeger) School of Mathematics and Statistics, The University of Western Australia, 35 Stirling Highway 6009 Crawley, Western Australia, Cheryl.Praeger@uwa.edu.au WWW.MATHS.UWA.EDU.AU/ PRAEGER

(C. Schneider) Departamento de Matemática, Instituto de Ciências Exatas, Universidade Federal de Minas Gerais, Av. Antônio Carlos 6627, Belo Horizonte, MG, Brazil, CSABA@MAT.UFMG.BR, WWW.MAT.UFMG.BR/ CCSABA 